\title{
Requirements on Integrated Information Systems from an Ecological Point of View
}

\author{
Lenz, R.J.M. \\ GSF-Research Centre for Environment and Health, PUC \\ P.O. Box 1129, 85758 Oberschleissheim, Germany, \\ Telefon: ++49-89-31872954, Fax.: + +49-89-31873369, \\ e-mail:lenz@gsf.de
}

\begin{abstract}
The paper intends to give some answers to the following three questions, related to the need of integrated information systems in ecological research: (i) what are the objectives and objects in ecology, (ii) what are the basic sources of electronically supported information in ecology, (iii) what are then the requirements and how could they be achieved? The first question will be answered in subdeviding the "world“ in levels of biological organisation of matter and environmental media, for which further classifications and clear definitions are urgently needed. Related to these deficits data and knowledge are still lacking. Besides that, more could be done out of the already existing information if integration and scaling approaches would be enhanced.

The sources for information are basically (relational) database management systems (DBMS), geographical information systems (GIS) and (dynamic) feedback models. In addition, user friendly and cheap data exchanges and accesses to the information systems are needed and to be widely available. Lacking up to now are flexible interfaces to connect the often decentral and specifically designed databases and information systems, and also sufficient metainformations. In giving some answers to the third question an environmental research information system (UFIS) will be described briefly. It is an operational information system for environmental models, and will be enlarged to data-descriptions in the near future.

Although there are still unsolved technical as well as logical problems, the main need of integrated information systems is the ecological information itself.
\end{abstract}

\section{Keywords}

ecological systems, integration, information system, ecological models 
The need of integrated information systems in ecological research is quite obvious if we agree in ecology as a new, integrative discipline in the sense of Odum (1977). He urged all the scientific specialities contributing to ecology to merge and integrate holistic and reductionistic approaches in order to meet the challenge of environmental protection. Because of the wide range of objectives of ecological concern one could be doubtful, whether this can ever be achieved. On the other hand, interpretations and applications of ecological knowledge increases drastically, and besides the economics ecology plays an important role in decision making - and thus feeds back ecological research.

To provide the information necessary, a sound basis of knowledge and data has to be elaborated, collected, stored, and retrieved for a broad community of researchers and environmentalists. Data highways are arising, forcing additionally ecology to provide and disseminate their information. Do we have these informations? Are we using the tools already available, and are they sufficient? In spite of the evidence of the need of integrated information systems the basis for that and therefore the reality is rather disappointing.

\section{RISING SOME QUESTIONS}

\subsection{What are the objectives and objects of ecology?}

Due to its youth and its wide range of objectives ecology is more diffuse than every other scientific discipline. It is even worse, because integration itself will be only accepted in the scientific community if there is a method behind. And up to now only mathematicians do have distinct rules and methods how to integrate. Odum (1977) didn't mention this kind of methodological lack of integration, but also nobody else.

Ecology deals with interrelations of biota and abiota, organising itself on various levels. These organisational levels of matter (cf. Miller 1975) can be observed, described (structurally) and analysed (functionally). As every part of our environment they are classified objects, integrating pattern and process, structure and function, holism and reductionism. The range of these organisational or integrative levels, the so-called realm of ecology, lasts from organisms to ecosystems and landscapes, or is even more extended. Fig. 1 shows these hierarchy in principle. These levels do have budgets and are closely linked to environmental media like organisms, soils, water, and air. These media are often the objectives of protection and research, but their quality and reactions cannot be understood without understanding the system they belong to. Hence the objectives of ecology are as well the levels of organisation and the corresponding media. Only in distinct and more or less simple cases we can disregard any interaction between them: cause-effects relationship like a shot leading to dead or clear injury are rather the exceptions than the actual impacts on ecological systems and their reactions.

In most cases, clarifying the complex cause-effect relationships is the challenge. Hence it is suggested, to regard three levels of integration in minimum at the same time, if one wants to detect changes or harmful effects of the one level in-between and its interrelation to environmental media (Lenz 1994).

But what does this simple statement really mean? Are these levels finally and operationally defined? And again, how to integrate empirical-descriptive knowledge with the analytical- 
quantitative one? How to interrelate the knowledge of neighbouring levels? They are mostly not well defined, especially the higher ones like ecosystems, the integration methods are still unclear or very case-specific, and scaling for interrelating the levels was just invented a few years ago!

I will give a few examples, mainly for the ecosystem level, starting with definitions:

An ecosystem is an entity of biotic and abiotic compartments, able to selforganize. Hence the minimum set of parameters describing the ecosystem includes living organisms and interactions with some abiotic environment. Based on this definition an ecosystem is somehow dimensionless and hard to classify. If you do not make further specifications, to my opinion you are lost, as well scientifically (for further analysis) as for application (what to protect?). This is one of the reasons, why Haber (1979) suggested, to embed the ecosystem between biocoenoses and landscapes, or instead, Ellenberg (1973) subdevided ecosystems itselfs hierarchically. But the classification systems are still provisional, and actually a lot of theoretical and task-specific approaches can be collected and discussed (Klijn 1994).

If one agreed in a classification system to be used, the problem of integration still remains. As mentioned before, integration has to deal with combining empirical-descriptive knowledge with analytical-quantitative ones. The original meaning is "to complete something“, hence integrative theories, or hypotheses and models have to be delineated from both of the approaches. For example models for forest growth: Statistical and descriptive models like the yield tables, based on the assumption of site constancy, have to be merged with the material balance equation, describing the processes at the site by including the concepts of electroneutrality and material turn over (Ulrich 1987). Both of these approaches alone are still hypotheses and do gain from combining and integrating them, although the process orientation and analysis was a great step forward to come to more reliable predictions.

Interrelating the integration levels means again, to integrate observation with process analysis. For instance, analytical experiments on a landscape level - e.g. changes of landuse patterns which can be related to higher sensitivities like erosional hazards or nitrate leaching do have their counterparts in observations on the ecosystem level - namely erosion or nitrate leaching! The parameters and models describing the interactions may and will be different at the two levels, but without understanding the landscape the ecosystem analysis will be insufficient because of its therefore limited parameters.

Integration by combining descriptive and process-oriented approaches, and scaling them to neighbouring levels above and below, will lead to more understanding. In addition to the integration defined above, scaling needs to reflect on the level-specific properties and indicate and interrelate them if appropriate.

To conclude these rough answering to the questions arised from ecology, we have to state that integration is a clear necessity but still an unclear method. It depends very much on the definitions of the objectives and the possible harmful effects to be described, analysed and modelled. Therefore the design of information systems must be very flexible and somehow preliminary. 


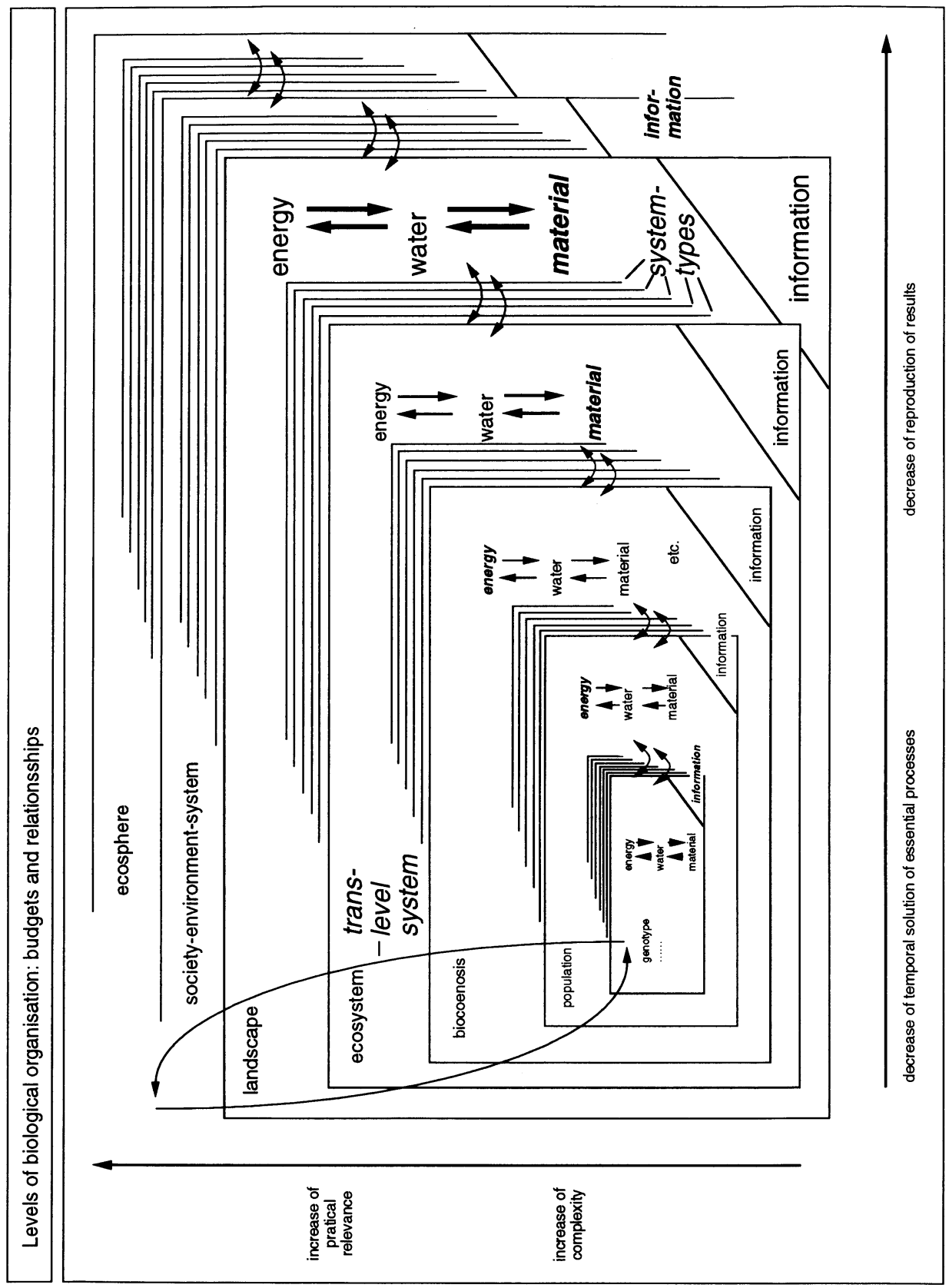

Figure 1 Organisational levels of matter. 


\subsection{What are the basic sources of electronically supported information in ecology?}

The basic sources of information - not only in ecological research - can be devided into three categories:

- point data (and its time series)

- spatial data (and its time series)

- models (to describe the knowledge e.g. at certain integration levels).

Ideally all these sources of information can be combined via flexible interfaces in order to ask for both, models and data, and how they can support each other.

Therefore all these categories do need a very sophisticated detection and description of their context, often called meta information or co-data.

The first category can be covered by database management systems (DBMS), which do provide storage capacities, querying and retrieval. In general their design should not be different for ecologically relevant data, than for other purposes. Nevertheless, the variety of data and the further need of interconnectability do make it necessary to have some conventions on hard- and software used, as well as clear meta-information. In addition, most of the ecological research is performed by non-computer specialists, which means, that user friendly interfaces have to be provided. The same holds true for the spatial data and the GIS and image processing systems. Of course, there are good systems available, but some of them need extensive software knowledge and/or money, and the equipment available for ecological research is far from being adequate concerning these constraints.

The situation becomes even worse, when we look at the facilities of the modelers actually under use. They use what they get, and hence often in very different hard- and software environments. Even the description and documentation is rather incomplete; no routines of plausibility and sensitivity checks are common. As a result the models constructed in most of the cases can be only understood and applied by the modelers itself.

\subsection{What are the requirements, derived from ecological needs and information systems actually used?}

If we do have to accept the wide range of objectives, the preliminary of classifying the objects, and the also wide range of information systems actually used, we are seriously in trouble.

On the other hand, knowledge and data, information technology and the demand of the scientific community as well as the decision makers for environmentally relevant information increases drastically. In addition there is a need on grading and evaluating these information, e.g. by setting up standards for documentation and meta-information, or even how to gain data and design models.

As a consequence the Ministry for education and research in Germany (BMBF) decided in 1992 to establish an environmental information system, which focuses on ecological research. Up to now the design of the project does not cover all of the aspects ideally to be regarded from an ecological point of view, but is already partly operational. Hence in the following we will give you some insight in the project called UFIS, which is the German acronym for Environmental Research Information System. 


\section{THE UFIS CONCEPT (SEE LENZ ET AL. 1994)}

One part of UFIS is designed to offer information on ecological models as a whole. The goal of UFIS is to provide a tool that supports interdisciplinary work among modellers as well as to give administrative bodies an overview over national and international activities in ecological modelling. The availability of such an information system is believed to be crucial to international co-ordination of modelling activities. Model comparisons and questions of model coupling have been defined as key goals in many IGBP activities (IGBP, 1994). Consequently UFIS will pay particular attention to data requirements of models, areas of application, and scales of the model.

The second part of UFIS which is closely linked to the model documentation focuses on the description of data. The interface to be established follows the items "need of data" and "data yield" in the model documentation. Hence in a final stage of development UFIS will consist of two meta data bases, one on models and one on data.

The background of archiving, describing and the retrieval of such information can be elucidated by reflecting within UFIS the pathways and stages necessary in developing models, and thus boiling down and concentrating the knowledge gained by scientific work. This should make sure that the context of a model - from data requirements to the characterisation of the ecological system considered - is documented within UFIS and therefore retrievable (figure 2). The reason that models and their respective data descriptions were chosen for documentation is that they represent condensed knowledge of complex situations. This does not hold for data, keywords, etc. alone. Here we use the term "model" in the widest sense, basically any kind of combination of data, even simple ones.

The method of modelling can be characterized by five mutually related "sources of information" (figure 2):

1. Conceptual models, i.e. verbal models, hypotheses and theories, strategies etc.. These are derived from observable reality as well as real data about reality (i.e. empirical and quantifying).

2. Mathematical simulation models: they represent the formal counterpart of conceptual models and allow similar as in an experiment to produce data that could be compared to reality. If successful, prognoses and transfer in areas not considered is possible.

3. Computed data,

4. measured data, and

5. observable reality (e.g. certain species and their overall behaviour)

have been mentioned already as required input to and output yielded by models (see above).

These five sources of information have their counterparts in the components of the information system UFIS: the retrieval system contains a user guidance by thesauri, systems ecological classifications and environmental media, thereby covering essential parts of the model concept. More detailed information reaching as far as the mathematical description of ecological processes is then accessible from the full model documentation which is available as a paperbased completed questionnaire as well as on-line from the database. This enables identification of data requirements of a model as well as data yield by the model. Note that UFIS contains only data- and model-descriptions (so called meta data), not data and models themselves. The data and models themselves usually relate to an ecological context, i. e. they permit conclusions only under certain ecological conditions. Basically UFIS contains five (or four, if 
one considers the retrieval system only as a means of access to ecological classification and model documentation) mutually related categories of information covered by the questionnaire. Therefore it is likely that ecological knowledge has been described in a transparent way and qualitatively verifiable.

The systems ecological classification has to cover all ecological systems from the cell (and its environment) up to the ecosphere. The general structure is based on a hierarchical concept of the organisation of matter (Miller 1975, Lenz 1994), on the one hand, and the environmental media (soil, water, air) on the other. Although "ecological" by definition counts for feedback of biotic and abiotic factors, a lot of so called ecological research is classical work in physics, chemistry or biology. Hence the keywording by disciplinary thesauri already in use might be sufficient. Therefore we decided to give further and more flexible orientation in UFIS also by just key-wording what is modelled while not disregarding the ecological classification above. Further descriptions of the context are budgets modelled (if applicable; e.g. water), geographical location, and environmental settings.

Besides the focus on the context of ecological knowledge documented by models and data another feature of UFIS is the flexible degree of details of the information in order to meet the needs of a wider range of users.

\subsection{The Model Questionnaire}

Model questionnaires have been developed already for specific purposes. There is e. g. one for forest models (Tiktak et al. 1993), and one for crop models (GCTE 1994). These references contain completed questionnaires of 18 and 12 models, respectively. A common feature is that these schemes are tailored for their specific area of application, and they cannot be applied to general ecological models. However, this does not apply to the "handbook of ecological models" (Jørgensen et al. 1994) on which work is currently in progress. This handbook will contain information on several hundred models. All these references give a good sample on the contents of the models but do not provide details like process descriptions. Also the information on the models is not available in a computerised database, but only in printed form. Therefore searches are convenient if one knows the names of the models one is looking for. A severe drawback of all questionnaires that we are aware of, is the freedom that they grant for making entries on it. Most entries are in free text, so the amount of detail given is up to the person who completes it. Furthermore this freedom undermines convenient searches with prescribed keywords. As a consequence we were confronted with the task to design a new questionnaire that is applicable to a wide variety of models keeping the intended use in a computerised database in mind.

The information contained in this scheme on a particular model is threefold: general information, description of processes within the model, and technical information. The first section provides general information on the model. Besides the obvious items like name and address of the modeller, a brief summary of the aim of the model and its basic ideas, valuable information regarding the range of applications is given. This includes references to other models that could possibly be coupled with the one under consideration. The suitability of the model for purposes other than the original one can to a certain extent be judged from reports on calibrations and validations performed (and the data been used) as well as spatial and temporal scales. References to user manuals and to a detailed model documentation are available as well. A flow diagram of the model structure will provide the UFIS user with an idea of the organisation of the model and its sub models. 
Methodical approach for the construction of models [Tob92]

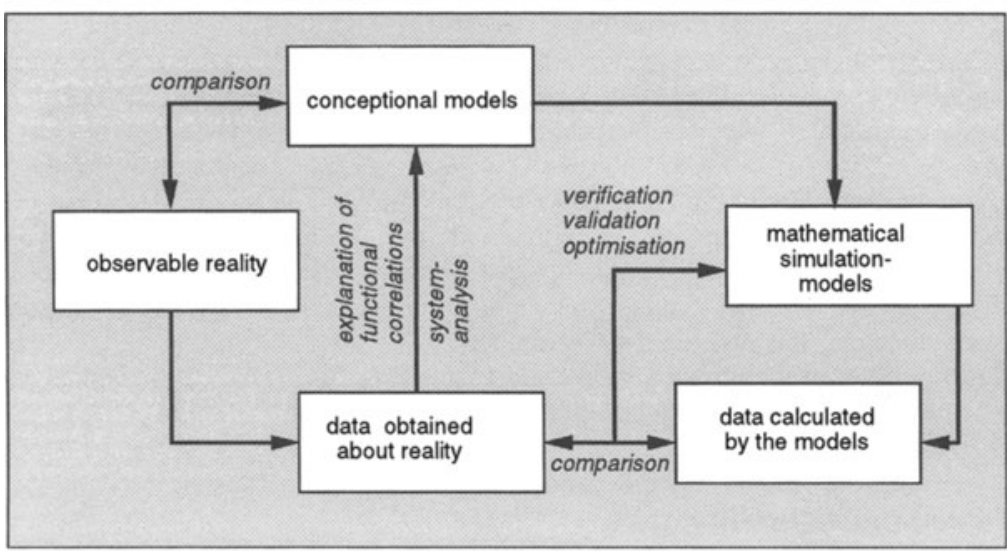

\section{Contents of an information-system about models and data in ecology}

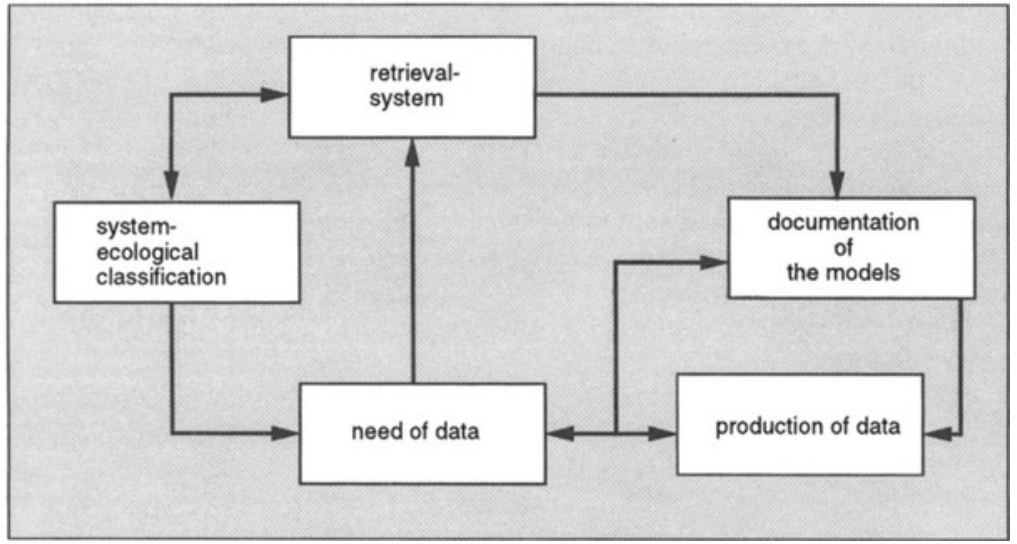

Figure 2 Concepts of modelling versus concepts of information systems. 
The second part of the scheme is influenced by the ideas to describe each ecological process and its realisation separately. As the hierarchy among the processes within the model is given by the coupling between these equations via variables information is stored for each process separately, referring to underlying subprocesses via pointers. This information includes for each process: brief description, mathematical formulation, possible constraints, initial/boundary conditions, spatial and temporal resolution, references to the literature and embedded subprocesses. A set of keywords regarding the type of process, type of equation, type of balance/flow enables an efficient retrieval. Quantities used in the mathematical formulation will be classified as input and output for each process, indicating also which quantities are input and output resp. to the full model. For each of the quantities a brief description, values used, range of ecologically relevant values is given and also the source of the data (where applicable). It is also reported on sensitivity analyses performed by the modeller. In case the mathematical description of a model cannot be split into different processes there is the option to describe the model verbally. However, we designed the structure of the scheme in a very general way, such that this case is considered the exception rather than the rule. For each single process a table of environmental conditions contains a description of its environment. Entries in this table describe the physical environment in which the underlying process formulation is valid. One of the items asked is the "level of organisation" (Miller 1975, Lenz 1993) on which the process takes place and a more specific characterisation within the chosen level (which type of ecosystem, which species etc.). Checking lists for these specifics are supplied; in particular a comprehensive thesaurus for types of ecosystems has been developed. Further items sought are geographic location, type of climate, and type of soil. The particulars are to be given as general as possible without violating the validity of the process formulation. Where meaningful and available, information will be given by means of commonly used classification schemes (e. g. the FAO-scheme for soil types).

The third section of the questionnaire deals with the computer implementation of the model. It is devided in software and hardware related information. Software questions covered are the possible requirement of certain libraries in order to run the model, programming language used and where to get complete program documentation. For the goal of UFIS as outlined above the data question is of particular importance. The UFIS user will find here detailed description of the input files needed and also where to obtain them from. Of course sources of the program code itself, demo input, and sample output files are also given. Hardware requirements are also covered, i. e. for which operating systems model code versions are available, as well as disk space required (Lenz et al. 1994).

\subsection{Technical Realisation of UFIS}

The goal of the UFIS database development is the coverage of all items from the model questionnaire and their storage in a relational database management system. Therefore the questionnaire represents the foundation of the database. The database is currently being implemented with the database management system Ingres (version 6.3) on a Convex super minicomputer. Usage of the database is possible with very modest hardware requirements via worldwide networks.

\section{Database design:}

An analysis of the model questionnaire reveals that it is a strongly structured text document. The items inside the boxes on the questionnaire can each be viewed as a separate page on its 
own. Within the boxes further subdivisions are possible. These pages together with their mutual relations represent the complete model documentation. Therefore it is natural to view the model documentation as a hypertext document; this is the underlying idea in the design of the database. In order to realise this idea there was in general one table created for each box on the questionnaire. Due to technical limitations some boxes required two tables. Additional tables were also required for the list of keywords and the system ecological classification as well as for the purpose of database administration.

\section{User interface:}

There are two user interfaces available for retrieval in the database. The first one is alphanumerically oriented and can be used with any ASCII-terminal. It offers two kind of screens, one for searching and one for browsing. The search screen allows the selection of a set of model documentations by prescribed conditions. The browsing screen provides views on the hypertext pages of the model documentation. Access is via X.25 or internet.

The second user interface is integrated in the world wide web (WWW), a world wide distributed hypertext system. The UFIS database is integrated into the WWW by allowing output of model information in HTML format (hypertext markup language) which is the standard format within the WWW. Access is via URL: http://www.gsf.de/UFIS/ufis.

In addition to the two interfaces for UFIS users, there is another interface for the UFIS managers for administration and input of data. For an illustration of the various interfaces see figure 3.

\section{UFIS Database}

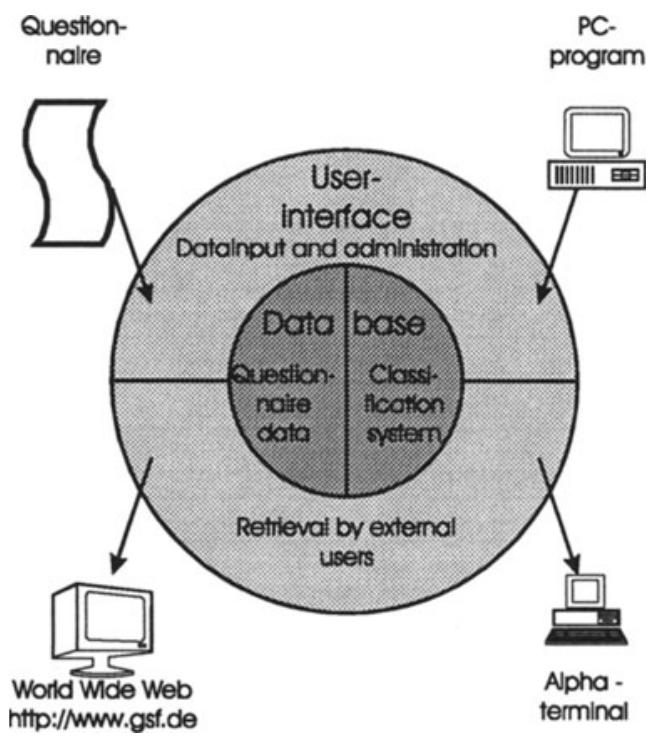

Figure 3 Access to UFIS. 


\title{
3.3 Perspectives
}

In this section we want to deal with the questions (i) how to choose models for documentation, (ii) what kind of further interfaces to other information systems should be developed, (iii) how to convince the modellers to document in UFIS, and (iv) how the availability of models and data itself could be improved. The best way to start with the model documentation is not to do this randomly but systematically. Due to the duties and goals UFIS, UFIS started out with BMBF projects and models, selected about 30 from the BMBF-managed database DAVOR, and about 120 by an inquiry at ecosystem research centres throughout Germany. In general future selections will follow the principles of representativeness which means a choice of a wide range of ecological models, both from different levels of organisation as well as more aggregated and more process-oriented ones. At present various information systems are under development, and in the near future a careful check of possible cooperations and interfaces has to be done. An intensive review of information systems in ecological research has been carried out. Also the meetings of UFIS and others supported further links of information systems for adapting the contents or technical features or both. Up to now it is planned to collaborate and possibly interface with the ecosystem research centres, the $\mathrm{PIK}^{1}, \mathrm{DKRZ}^{2}, \mathrm{NLB}^{3}, \mathrm{BGR}^{4}$, $\mathrm{DLR}^{5}, \mathrm{UNEP}^{\mathrm{HEEM}}{ }^{6}, \mathrm{GKSS}^{7}, \mathrm{ZADI}^{8}, \mathrm{KfK}^{9}, \mathrm{UFZ}^{10}$ and the University of Kassel. The world wide web (WWW) will be the tool for most of these links, but hopefully the documentation scheme will be adopted by the institutions themselves, and many modellers will feed the UFIS database with only little external support. In order to convince others of UFIS and ensure broad support a number of meetings and presentations has been scheduled. Also strong support from the sponsoring agencies is necessary. As can be seen from many other examples scientists and modellers themselves should adopt the concepts and carry out input work with the support by two measures:

- the documentation is part of the project itself ("forcing" the modeller)

- the documentation is widely accepted by the scientific community and leads to closer contacts with other scientists and ecological planners in these fields.

While the first kind of measure is a task of the sponsoring agencies, the second one is ours. Hence there is a need for permanent staff to support the modellers, update and represent the projects.

To improve the availability of models and data themselves is one of the major tasks for the future. We have to come to active libraries of models and data. Starting with the meta-level has the advantage to allow to specify the actual needs and gives a comprehensive overview on

\author{
Potsdam Institute for Climate Impact Research \\ German Climate Computing Centre, Hamburg \\ Geological Survey of Lower Saxony \\ Federal Institute for Geosciences and Natural Resources \\ German Aerospace Research Establishment \\ UN Environmental Programme - Office for Harmonization of Environmental Measurement \\ Research Centre Geesthacht \\ Centre for Agricultural Documentation and Information \\ Nuclear Research Centre Karlsruhe \\ 0 Environmental Research Institute Leipzig - Halle
}


models and data that would otherwise possibly remain widely unknown. We should use this advantage for the selection and structuring of true model- and data-bases.

\section{CONCLUSIONS}

Let me finish my presentation with an example, summarising the needs for integrated information systems. Information is by definition never a single data, its a difference between in minimum two data, may be more, and my be varying ones; and the best information is knowing all the „differences“ and contextual data:

\section{Example}

5

$5 \mathrm{~kg}$

$5 \mathrm{~kg}$ apples

$5 \mathrm{~kg} \quad$ of one tree

",$\quad$ per year

measured with ...

$<$ the average yield

but premium quality

do have a prize of ...>

and: Yield was low because of frost during blooming; growth development can be modelled with the following variables ... but model is only valid for certain environmental conditions and does not include possible pest attacks.

From this example it might be obvious, that neither one database nor a few datas and models are able to cover the field of interest.

\section{REFERENCES}

Ellenberg H. (1973): Versuch einer Klassifikation der Ökosysteme nach funktionalen Gesichtspunkten. In Ellenberg, Hrsg., Ökosystemforschung, Springer, 235-266.

GCTE (1994): Focus 3 Wheat Network: 1993 model and experimental meta data. GCTE Report, 2, Canberra.

Haber W. (1979): Theoretische Anmerkungen zur ökologischen Planung. Verh. Ges. Ökol. J., 19-30.

IGBP (1994): IGBP Global Modelling and Data Activities 1994-1998. IGBP Report, 30, Stockholm, 1994.

Jørgensen S. E., S. Nors Nielsen, and B. Halling-Sorensen (1995): Handbook of Environmental and Ecological Models. CRC Press, to appear.

Klijn, F. (ed.) (1994): Ecosystem Classification for Environmental Management. Kluwer Academic Publ., Ecology and Environment, Vol. 2, 293. 
Lenz R. (1993): Systemökologische Anforderungen an ein Umweltforschungsinformationssystem (UFIS) als operationale Basis für Scaling - Konzepte in der Umweltplanung. In A. Jaeschke, T. Kampke, B. Page, and F. J. Radermacher, editors, Informatik für den Umweltschutz, 7. Symposium Ulm, Springer, 427- 440.

Lenz R. (1994): Ecosystem classification by budget of material; the example of forest ecosystems classified as proton budget types. In F. Klijn, editor, Ecosystem classification for environmental management, Kluwer, 117-137.

Lenz R., M. Knorrenschild, C. Herderich, O. Springstubbe, E. Forster, J. Benz, M. Asshoff, W. Windhorst (1994): An information system of ecological models. GSF-Bericht 27/94, $92 \mathrm{~S}$.

Miller G. T. (1975): Living in the Environment - Concepts, Problems, and Alternatives. Wadsworth Publ.

Odum E.P. (1977): The emergence of ecology as new integrative discipline. Science 195 (4284), 1293-1298.

Tiktak A. and J. M. van Grinsven (eds.) (1993): Model questionnaires. Handout at the workshop on comparison of forest-soil-atmosphere models, Leusden.

Ulrich B. (1987): Stability, elasticity and resilience of terrestrial ecosystems with respect to matter balance. In Schulze E.-D. Zwölfer H., editors, Potentials and limitations of ecosystem analysis, Ecol. Studies, Vol. 61, New York, Springer, 11-49.

\section{BIOGRAPHY}

\section{Education and Degrees}

- Ph.D. ("Dr. agr.", summa cum laude) in Agriculture, Munich University of Technology, Germany, August 1991. Thesis: "Characteristics and Loads of Forest Ecosystems in Northeastern Bavaria - a landscape-ecological Evaluation based on materieal budgets" (in German), supervised by Prof. W. Haber and Prof. B. Ulrich

- ("Diplom", summa cum laude) in Agrobiology, Hohenheim University, Germany, June 1982. Thesis: "The impact of wildlife on ground vegetation in forests" (in German), supervised by Prof. J. Pfadenhauer

\section{Experience}

- since Sept. 1992: Working group leader "Information Systems for Environmental Research and Planning", altogether about 12 scientific coworkers at the GSF-Research Centre for Environmental and Health

- Jan. 1990 - Aug. 1992: Assistant Professor at the Chair for Landscape Ecology (Prof. W. Haber), Munich University of Technology, Germany

- Nov. 1985 - Oct. 1989: Research scientist at the Chair for Landscape Ecology 